\title{
Évaluer la compétence par un test de jugement de grammaticalité : le cas de la relativisation chez des apprenants chypriotes hellénophones avancés en FLE
}

\author{
Monique Monville-Burston \\ Université de Chypre \\ mburston@ucy.ac.cy
}

\section{Introduction}

Le test discuté dans cet article a été élaboré pour tenter de décrire plus précisément et de mieux comprendre l'interlangue d'étudiants chypriotes grecs avancés en français langue étrangère (FLE), dans le domaine de la relativisation. Il s'agit d'un test de jugement de grammaticalité (TJG), c'est-à-dire d'une tâche dont le but est d'inférer les règles syntaxiques qui font partie de la compétence langagière des apprenants, plutôt que d'observer leur performance (au sens chomskyien des deux termes). Les résultats de l'étude auront des aspects méthodologiques, puisque le test dans son état actuel sera soumis à un examen critique. Ils seront aussi descriptifs : on dégagera des traits importants de l'interlangue de sujets dont l'appropriation du FLE a jusqu'ici été peu étudiée. Cela se fera dans un domaine (la relativisation) sur lequel, au contraire, on a déjà beaucoup enquêté pour des binômes autres que grec/L1-français /L2 (par exemple Doughty 1991 ; Eckman et al. 1988 ; Hancock \& Kirchmeyer 2002, 2005 ; Hawkins 1989 ; etc.). L'étude aura aussi des implications théoriques du point de vue de l'appropriation de la syntaxe de la relativisation: on s'interrogera d'une part sur la portée des transferts (Gass 1979), de la grammaire universelle (Hawkins J. 2007), de l'extension de la hiérarchie typologique d'accessibilité de Keenan et Comrie (1977) au domaine de l'acquisition des relatives en L2 (Eckman et al. 2007 ; Hawkins R. 2007 ; Izumi 2003); et d'autre part sur l'influence de la situation particulière d'apprentissage, celui-ci se faisant en milieu diglossique (dialecte chypriote-grec standard) et non pas monolingue.

Dans cette présentation, nous examinerons d'abord, comme point de référence, l'utilisation des pronoms relatifs dans les productions écrites libres des sujets qui ont participé à l'expérience. Suivra une discussion de la méthodologie choisie, à la lumière des travaux déjà effectués sur la validité et la fiabilité des jugements métalinguistiques des apprenants dans l'évaluation de leur appropriation d'une L2. Finalement nous présenterons et discuterons les résultats obtenus les plus marquants.

\section{Contexte sociolinguistique et faits linguistiques}

La relativisation est un domaine de la syntaxe française qui jusqu'en dernière année d'études universitaires (niveau Master 1) est source d'entorses variées à la grammaire standard chez les étudiants chypriotes hellénophones de FLE. À titre d'exemple, considérez les phrases reproduites en (1) :

(1) (a) une politique *que $<=$ qui $>$ faisait de l'anglais la langue officielle

(b) nous *que nous $<=$ qui $>$ ne parlons pas créole

(c) des écrivains que leur $<=$ dont la $>$ vocation [...] réside dans une croisade

L'une des raisons de cette situation -parmi d'autres qui restent encore à déterminer-c'est sans doute la nature du pronom relatif dans les trois langues/variétés qui sont en présence lors de l'apprentissage. Dans le dialecte grec chypriote, le relatif est une particule $\pi$ ov (pou), qui sert par ailleurs aussi de conjonction de subordination (équivalente de que) après certains types de verbes. Sa forme invariable ne permet pas de lui assigner des marques de genre, de nombre et de cas. Ce relativiseur-complémentiseur polyvalent 
sert aussi d'adverbe relatif de lieu et de temps. Il peut en outre tenir lieu de syntagme prépositionnel relativisé, comme on le voit dans (2).

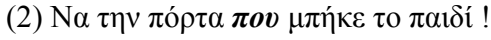
Voici la porte par laquelle l'enfant est entré !

On retrouve ce $\pi o v$ en grec standard, langue utilisée par les Chypriotes grecs comme variété haute dans leur diglossie ${ }^{1}$. Cependant il existe aussi pour le grec soutenu un autre relatif $о$ олоіо ( contrairement à $\pi o v$ est fléchi en genre, nombre et cas, et qui dans les syntagmes prépositionnels est introduit par la préposition requise par le sens ${ }^{2}$.

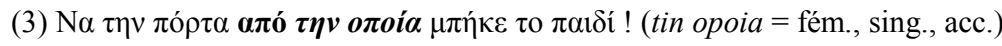

Voici la porte par laquelle l'enfant est entré !

Quant à la langue cible, le français, le relatif y varie parfois en cas [qui/que/dont] et parfois en genre et en nombre [lequel/laquelle/lesquel(le)s], ce dernier pronom étant normalement introduit par une préposition ${ }^{3}$. S'ajoutent à ces formes l'adverbe relatif de lieu/temps où et le pronom 'neutre' ('collectif') quoi. Le Tableau 1 résume schématiquement les différences entre les trois systèmes ${ }^{5}$.

\begin{tabular}{|c|c|c|c|}
\hline $\begin{array}{cc}\text { Langue/variété } & \rightarrow \\
\text { Fonction } & \\
\text { grammaticale } & \\
\downarrow & \end{array}$ & $\begin{array}{l}\text { Grec chypriote et grec } \\
\text { commun - variété } \\
\text { basse dans la diglossie } \\
\text { chypriote } \\
\text { Système (1) }\end{array}$ & $\begin{array}{c}\text { Grec commun soutenu } \\
\text { - variété haute dans la } \\
\text { diglossie chypriote } \\
\text { Système (2) }\end{array}$ & Français normalisé \\
\hline Sujet & $\pi o v$ & $\begin{array}{c}\text { o oлoíos (fléchi en } \\
\text { genre, nombre et cas) }\end{array}$ & $q u i$ (marque le cas) \\
\hline Objet direct & $\pi o v$ & $\begin{array}{c}\text { o o } о \text { oíos (fléchi en } \\
\text { genre, nombre et cas) }\end{array}$ & que (marque le cas) \\
\hline \multirow{2}{*}{$\begin{array}{c}\text { Syntagme } \\
\text { prépositionnel }\end{array}$} & \multirow[t]{2}{*}{$\pi o v$} & \multirow{2}{*}{$\begin{array}{c}\text { Prép }+ \text { o o } \text { oiós (fléchi } \\
\text { en genre, nombre et } \\
\text { cas) }\end{array}$} & dont (marque le cas) \\
\hline & & & $\begin{array}{c}\text { Prép. + qui ou Prép. }+ \\
\text { lequel ou Prép. + quoi } \\
\text { (marquent genre et } \\
\text { nombre et/ou } \\
\text { l'opposition +/- } \\
\text { humain, et/ou } \\
\text { l'opposition +/- } \\
\text { collectif neutre) }\end{array}$ \\
\hline Adverbe relatif & $\pi o v$ & ó & où \\
\hline
\end{tabular}

Tableau 1 - Comparaison des formes du relatif: grec chypriote/grec commun; grec commun soutenu; français normalisé

Exception faite de l'adverbe relatif, du point de vue de la flexion morphosyntaxique, le système (1) est sous-spécifié comparé au français normalisé, tandis que le système (2) est sur-spécifié puisqu'il marque dans tout emploi, le genre, le nombre et le cas. Le système (3) est complexe et hybride ${ }^{6}:$ il marque parfois le cas (et marginalement l'opposition $+/$ - humain, ou l'opposition $+/$ - collectif neutre) et parfois le genre et le nombre.

Il est important de noter que, dans le système vernaculaire (1), des pronoms clitiques de reprise (résomptifs), qui marquent le cas, peuvent être présents dans la proposition relative. Ces constructions analytiques, avec décumul des fonctions de démarcation et d'anaphoricité, sont obligatoires pour les possesseurs, fréquents pour les objets indirects, facultatifs pour les objets directs, mais impossibles pour les sujets (Mackridge 1985 : 249 ; Nicholas 1998), ce qui suit grosso modo la hiérarchie d'accessibilité 
(HA) de Keenan et Comrie. L'exemple (4) illustre une relative résomptive avec clitique en fonction d'objet indirect.

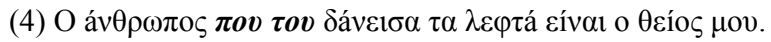

L'homme [mot-à-mot : que je lui ai prêté] à qui j'ai prêté l'argent est mon oncle.

Dans la perspective de l'enseignant, on peut envisager les effets de la L1 sur l'interlangue des apprenants en matière de relativisation de deux points de vue ${ }^{7}$ : du point de vue de l'effcacité de la communication et de celui de la stigmatisation qui peut résulter des erreurs. Dans le premier cas, les erreurs ne sont pas si graves qu'elles interfèrent dans les échanges verbaux, les étudiants avancés s'exprimant couramment. Les Français ont d'ailleurs l'habitude dans la conversation quotidienne d'entendre et d'utiliser des relatives non-standard, qu'elles soient réduites, résomptives, ou pléonastiques (Gadet 2003). Mais d'autre part l'utilisation de ces sortes de relatives peut résulter en un jugement défavorable porté sur l'étudiant étranger qui n'est pas supposé s'aventurer dans le français populaire.

L'étude que nous allons discuter, qui se trouve dans sa première phase, concerne des étudiants de quatrième année qui se spécialisent en français à l'Université de Chypre. Ce sont des apprenantsutilisateurs de FLE qui se situent au niveau B2-C1, selon les normes du Cadre Européen Commun de Référence pour les langues (CECR).

Comme point de repère nous avons d'abord examiné l'utilisation des pronoms relatifs faite par nos sujets en production libre. Nous avons analysé des travaux écrits, plus précisément un devoir de 600 mots environ en 2006 (20 étudiants) ; un partiel et deux devoirs de 600 mots en 2007 (12 étudiants). Ce corpus d'apprenants a montré les types et fréquences d'emplois des pronoms relatifs notés dans le Tableau 2.

\begin{tabular}{|c|c|c|c|}
\hline RELATIF & Nb total d'items & Nb d'items corrects & Nb d'items incorrects \\
\hline qui & 319 & 302 & 17 \\
\hline $\begin{array}{c}\text { lequel (hors syntagme } \\
\text { prépositionnel) }\end{array}$ & 5 & 0 & 5 \\
\hline que & 154 & 135 & 0 \\
\hline dont & 4 & 4 & 3 \\
\hline $\begin{array}{c}\text { qui (en syntagme } \\
\text { prépositionnel) }\end{array}$ & 2 & 23 & 3 \\
\hline $\begin{array}{c}\text { lequel (en syntagme } \\
\text { prépositionnel) }\end{array}$ & 26 & 54 & Total : $47(8,3 \%)$ \\
\hline où & 57 & Total : $520(91,7 \%)$ & \\
\hline
\end{tabular}

Tableau 2 - Pronoms relatifs en production libre (Étudiants de 4ème année, $N=32$ )

Le nombre d'erreurs est relativement faible, si on compare ces données aux données recueillies dans des travaux d'étudiants moins avancés, par exemple en $3^{\text {ème }}$ année : $15 \%$ de relatives erronées en 2006 et $12 \%$ en 2007. Les fréquences d'utilisation de relatifs cependant sont intéressantes. On remarque un grand nombre de qui (en fonction de sujet), un nombre important de que et une faible représentation des autres relatifs. Dont et qui (en syntagme prépositionnel) sont rares. De ce point de vue, il existe des similarités avec l'usage des pronoms relatifs chez les francophones (cf. les deux corpus cités dans Gadet 2003) ${ }^{8}$.

Les erreurs principales sur que viennent du fait que cette forme est traitée comme un relatif général, semblable à pou. Que est conçu comme une particule invariable qui opère pour toutes les fonctions grammaticales, ou dont la souplesse permet de mettre tout simplement en rapport l'antécédent et le contenu de la relative ${ }^{9}$, comme on le voit en (5).

(5) (a) *La classe, les professeurs, les apprenants sont cet environnement que $<=q u i>$ nous aide à apprendre.

(b) *[Ce] sont des procédures propres à la didactique qu'elles $<=q u i>$ s'appliquent 
On note aussi des confusions entre qui et que, dont certaines laissent entrevoir l'influence de la phonologie, comme en (6). Les déviances liées à lequel hors syntagme prépositionnel seront traitées dans la Section 4.4.

(6) *Le professeur doit utiliser des façons différentes [...] pour arriver au résultat qui $<=q u$ 'il $>$ s'est fixé.

On pourrait se demander si les relatives réduites (relatives en que, au sens de Gadet 2003) et résomptives du français non standard pourraient être incriminées dans la production de phrases telles que celles de (5a, b, c). Cela est douteux puisque nos étudiants sont peu exposés à cette variété de français et que ces types de relatives sont présents très tôt dans leur interlangue, à savoir dès le lycée, avant des séjours à l'étranger. Cependant c'est une variable qui mériterait considération, du moins pour les étudiants qui partent en échange Érasmus. Il faudrait voir si le semestre passé dans un pays francophone (en $3^{\text {ème }}$ ou $4^{\text {ème }}$ année), et donc l'exposition à des modèles qui convergent avec certains traits du système (1) et bien ancrés dans l'interlangue, contribue ou non à renforcer l'utilisation de relatives réduites ou résomptives.

Le Tableau 2 a fait apparaître l'une des faiblesses du corpus actuel, à savoir la représentation très limitée de certains relatifs. La production naturelle des sujets ne donne pas une image exhaustive de leur interlangue. Nous avons donc préparé deux outils expérimentaux pour recueillir des données supplémentaires : un TJG et un test de traduction ${ }^{10}$. Nous ne discuterons ici que le premier.

\section{Le test de jugement de grammaticalité}

\subsection{Procédure}

Le TJG contient 30 items, 15 corrects et 15 incorrects (cf. Tableau 3). Il a été administré à deux groupes d'étudiants de $4^{\text {ème }}$ année $\left(\mathrm{N}_{1}=20\right.$ et $\mathrm{N}_{2}=12$, soit 32 sujets en tout), de niveau B2-C1 selon les critères du CECR. Les items à juger ont été lus à haute voix par l'administrateur du test et les sujets avaient 12 secondes pour décider si la partie de la phrase soulignée (la proposition relative) était selon eux (1) correcte, (2) probablement correcte, (3) probablement incorrecte ou (4) incorrecte et, dans les deux derniers cas, pour rectifier la phrase, ce qu'ils n'ont pas toujours fait. Cette gamme de choix permettait de faire la différence entre jugement de grammaticalité et jugement d'acceptabilité. Les items choisis ont été tirés ou adaptés de phrases écrites ou dites par des étudiants d'années précédentes. Le relatif quoi et les relatifs précédés du démonstratif $c e$ n'ont pas été inclus. Les processus de relativisation sur lesquels nous avons sollicité le plus de jugements ont été la réductivité (c'est-à-dire la relativisation par simple connecteur, y compris pour les syntagmes prépositionnels) et la résomption. Enfin, il est important de remarquer que toutes les structures que contient le TJG ont fait l'objet d'un enseignement explicite dans les cours de grammaire et de langue de $1^{\text {ère }}$ et $2^{\text {ème }}$ années; il ne teste donc pas purement des traits de la relativisation propres à la grammaire universelle (Mandell $1999: 77$ ).

Nos hypothèses étaient les suivantes : a) Le TJG donnerait sur la compétence des sujets des renseignements rares ou absents dans les productions libres, où les auteurs peuvent contourner les zones de la relativisation qu'ils considèrent complexes ou difficiles; b) plus précisément, dans les cas d'incertitude, les sujets seraient plus tolérants de formes erronées qui correspondraient aux structures de leur L1 et par contre plus disposés à amender des formes correctes qui différeraient des structures de leur L1. 


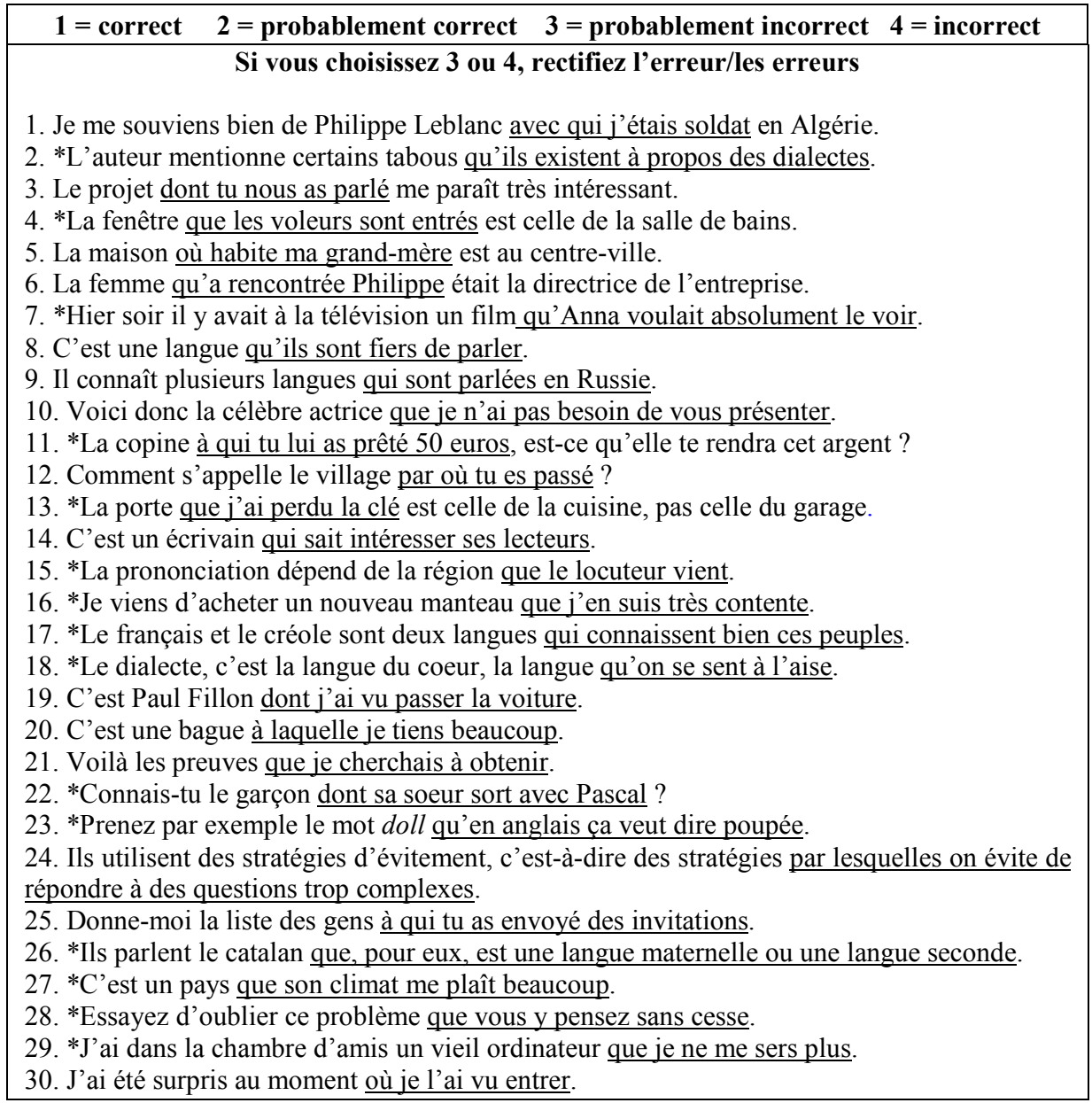

Tableau 3 - Test de jugement de grammaticalité (TJG) - Relativisation

\subsection{Considérations méthodologiques}

On connaît les débats qui ont entouré la valeur, la construction et l'administration des TJG. Rappelons qu'on s'est interrogé sur la validité de ces tests comme mesure de compétence dans une L2. Ellis (1991) compare les jugements métalinguistiques en L1 et L2 et recommande que les TJG en L2 soient validés avec soin avant qu'on puisse prétendre qu'ils reflètent fidèlement les habiletés langagières des apprenants. Il remarque en outre qu'on sait relativement peu de choses sur l'effet précis de facteurs comme le niveau de développement langagier des sujets, les structures grammaticales examinées et la nature du test. Sur la base d'une tâche d'évaluation comportant des réflexions faites à voix haute, Ellis établit aussi un inventaire des stratégies qui semblent caractériser la façon dont les apprenants d'une L2 portent leurs jugements. Dans la même ligne, Davies \& Kaplan (1996) montrent que les stratégies utilisées dans les TLG sont différentes et plus variées chez les apprenants de L2, comparés avec les locuteurs natifs.

Gass (1994) et Johnson et al. (1996) font de la fiabilité des TJG l'objet de leur recherche, compte tenu de la nature incomplète du savoir des apprenants ou utilisateurs non-natifs. Grâce à la mise en parallèle des résultats de deux administrations successives du même TJG, Gass démontre que le taux de variation dans les réponses est plus élevé (donc moins fiable), d'une administration à l'autre, pour les items où il y a le plus d'incertitude. D'autre part, en comparant la performance d'apprenants chinois adultes d'anglais et de locuteurs natifs d'anglais sur un TJG, Johnson et al. (1996) ont établi que les jugements des deux groupes 
différaient quantitativement -comme on s'y attendrait- mais aussi qualitativement, les premiers manifestant un degré élevé d'inconsistance dans leurs jugements de grammaticalité. Les auteurs émettent l'hypothèse que le 'savoir' des apprenants est fondé conjointement sur leur connaissance de la L2, sur des règles qu'ils ont fabriquées à leur propre usage ainsi que sur la décision de 'deviner' ou de répondre au hasard.

Tout en étant avertie de ces critiques et consciente des limites des TLG en L2, nous avons cependant opté pour l'utilisation d'un TJG pour les raisons suivantes :

- Comme nous l'avons dit plus haut, les productions libres de nos sujets ne nous donnaient pas accès à certaines zones du savoir déclaratif qu'ils avaient de la relativisation en français. Les devoirs écrits du corpus offraient essentiellement des instances de qui (hors syntagme prépositionnel) et de que.

- Nous pouvions espérer qu'il n'y aurait pas trop de variabilité dans les réponses, les étudiants étant dans leur dernière année d'études françaises et ayant une bonne connaissance de la langue. Nous avons supposé avec Davies et Kaplan (1998: 199) qu'à mesure que la compétence langagière en L2 se développe, les TJG fournissent des données plus valides et plus fiables ${ }^{11}$. En réalité, un petit nombre d'items (voir plus bas) ont entraîné une grande variabilité dans les jugements.

- Vu leur expérience du français, tout donnait à penser que les sujets auraient des intuitions (en plus de la connaissance explicite de règles spécifiques) qui leur permettraient de décider immédiatement et dans un temps de délibération limité (quelques secondes), de l'acceptabilité ou de la grammaticalité d'une phrase, et en cela se rapprocheraient des locuteurs natifs dans leur façon de porter des jugements métalinguistiques. On testerait donc en principe plus leur compétence que leur performance.

- Les TJG tendent à être validés par des mesures de performance faites à l'aide d'autres instruments (Chaudron $19: 371$ ).

Le design expérimental de notre TJG a été élaboré avec autant de rigueur que possible, en tenant compte des conseils et remarques méthodologiques de travaux comme ceux de Chaudron (1983), Ellis (1991) et Cowan et Hatasa (1994). Pour limiter la variabilité, un certain nombre de précautions ont été prises. Les réponses de type (2) < probablement correct> et (3) < probablement incorrect> permettaient aux sujets de nuancer leur jugement, si nécessaire, et d'opter pour des structures possibles ou qu'ils croyaient acceptables plutôt qu'absolument grammaticales ou agrammaticales. Les items qui ont servi de stimuli représentaient des cas clairs de phrases grammaticales et non-grammaticales, selon le français normé. La phrase 8 (C'est une langue qu'ils sont fiers de parler), cependant, s'est révélée être un item spécieux, puisque quelques étudiants l'ont jugée incorrecte et lui ont substitué la phrase, elle aussi correcte : $C$ 'est une langue dont ils sont fiers de parler. On sait que la longueur de la phrase et le choix du vocabulaire peuvent être des sources de variabilité. Nous avons veillé à ce que cette longueur soit raisonnable et, en adaptant des phrases produites par des étudiants d'années moins avancées, à ce que le vocabulaire soit usuel ou connu des sujets.

\subsection{Résultats généraux et discussion}

Ce test (comme tous les tests de son genre) ne nous a pas permis de savoir quelles stratégies les sujets utilisaient pour arriver à une décision (traduire, deviner, appliquer consciemment une règle acquise, appliquer une règle plus ou moins bien comprise, etc.). Il serait bien sûr utile et intéressant de connaître les processus cognitifs grâce auxquels les sujets ont formé leurs jugements (voir Ellis 1991 : 181 et Goss et al. 1994). Pour ce faire -et le projet est en cours de réalisation-, on peut obtenir des renseignements introspectifs de la part des sujets en les faisant exprimer à haute voix et en enregistrant les réflexions qui orientent leur décision.

Il est difficile de dire avec certitude si les jugements des sujets d'un TJG sont stables, si on ne procède pas à une seconde administration du même test Gass (1994). C'est ce que nous avons fait pour le second groupe d'étudiants testés $(\mathrm{N}=9)^{12}$. Les premiers résultats globaux sont donnés dans le Tableau 4 . 


\begin{tabular}{|l|l|l|}
\hline \multicolumn{1}{|c|}{ Types de jugements } & \multicolumn{1}{c|}{$\begin{array}{c}\text { Nb d'items jugés } \\
\text { (Total =30 x 9=270) }\end{array}$} & \multicolumn{1}{c|}{ Pourcentage } \\
\hline $\begin{array}{l}\text { Jugements identiques pour les deux séances } \\
\text { (Types 1-1, 2-2, 3-3, 4-4) }\end{array}$ & 127 & $47 \%$ \\
\hline $\begin{array}{l}\text { Jugements similaires pour les deux séances } \\
\text { (Types 1-2, 3-4) }\end{array}$ & 71 & $26,3 \%$ \\
\hline $\begin{array}{l}\text { Jugements identiques et similaires pour les deux } \\
\text { séances combinés }\end{array}$ & 198 & \multicolumn{2}{|c|}{$73,5 \%$} \\
\hline $\begin{array}{l}\text { Jugements opposés pour les deux séances } \\
\text { (Types 1-2, 2-3, 1-4, 2-4) }\end{array}$ & 72 & $26.7 \%$ \\
\hline
\end{tabular}

Tableau 4 - Variabilité dans les jugements (entre séance 1 et séance 2 du TJG $(N=9)$

$47 \%$ des réponses données par les 9 étudiants ayant participé aux deux séances indiquent des jugements identiques ; $26 \%$ des réponses sont similaires (la phrase est correcte -jugement 1 ou 2-/la phrase est incorrecte -jugement 3 ou 4-). Si on combine ces deux on remarque une certaine constance dans les jugements du groupe en général $(73,5 \%)$. Cela est encourageant pour la fiabilité du TJG. Cependant le nombre de sujets était trop bas pour qu'on soit entièrement positif ${ }^{13}$.

Contrairement aux sujets d'Ellis (1991) qui avaient rarement sélectionné «pas sûr » dans la gamme des choix proposée, un bon nombre de nos étudiants ont fait usage des options 2 et 3 <probablement correct; probablement incorrect $>$ dans leurs réponses : $23 \%$ pour le groupe 1 et $30 \%$ pour le groupe 2 ). Ils n'ont donc pas craint de laisser paraitre leur incertitude. En comparaison, l'étudiante française en échange Erasmus qui a servi de sujet témoin a donné pour tous les items des jugements tranchés : (1) correct ou (4) incorrect.

Nous ne savons pas non plus si une grande variabilité observée pour certains items (voir plus bas) reflète un manque de connaissance du point de grammaire considéré, ou si elle est due à certains traits de la phrase à juger qui ont pu distraire ou troubler les sujets. Des indices permettent de supposer, par exemple, que tenir à (phrase 20) n'était pas une expression avec laquelle tous les sujets étaient entièrement à l'aise. En outre quelques phrases ont été condamnées et corrigées par certains sujets sur la base de facteurs autres que la relativisation (temps verbal, accords, ordre des mots).

Pour plus de deux tiers des items (22 sur 30), l'ensemble du groupe $(\mathrm{N}=32)$ a globalement concordé dans ses jugements avec l'exactitude ou l'inexactitude d'un item (voir Tableau 5). Il est à noter que dans ce tableau, les réponses de type (1) et (2) d'une part, et (3) et (4) d'autre part, ont été regroupées respectivement comme $<$ items jugés corrects $>$ et $<$ items jugés incorrects $>$.

\begin{tabular}{|lc|ll|}
\hline Items corrects proposés dans le TJG : & 15 & Items incorrects proposés dans le TJG: & 15 \\
\hline Items jugés corrects par le groupe : & 10 & Items jugés incorrects par le groupe : & 12 \\
\hline Items jugés incorrects à tort) par le groupe $: 3$ & Items jugés corrects (à tort) par le groupe : & 2 \\
\hline Opinion également partagée : & 2 & Opinion également partagée : & 1 \\
\hline
\end{tabular}

Tableau 5 - Items corrects ou incorrects selon la norme et selon l'ensemble des sujets $(N=32)$

Les items qui font exception à cette tendance sont les phrases ci-dessous.

1. Je me souviens bien de Philippe Leblanc avec qui j'étais soldat en Algérie.

6. La femme qu'a rencontrée Philippe était la directrice de l'entreprise.

12. Comment s'appelle le village par où tu es passé ?

18. *Le dialecte, c'est la langue du coeur, la langue qu'on se sent à l'aise.

19. C'est Paul Fillon dont j'ai vu passer la voiture.

22. *Connais-tu le garçon dont sa soeur sort avec Pascal ?

23. *Prenez par exemple le mot doll qu'en anglais ça veut dire poupée.

25. Donne-moi la liste des gens à qui tu as envoyé des invitations. 
La moitié des sujets optent pour 'correct' et l'autre pour 'incorrect' pour les items 1, 12, 22 : l'opinion est également divisée. Par contre pour les autres items $6,18,19,23$ et 25, nous avons un jugement général inverse de ce qu'est la norme : un jugement d'acceptation ('correct') quand il aurait dû être de rejet ('incorrect') et vice versa. Nos sujets ne se sont donc pas comportés comme des locuteurs natifs. L'étudiante Érasmus, qui a partout été en accord avec la norme, en témoigne.

A quoi tient cette situation ? Certainement, dans certains cas, à la difficulté d'analyser la phrase proposée et de lui assigner un sens. L'inversion verbe-sujet de la phrase 6 a obscurci la sémantique et quand l'item a été corrigé par les sujets qui l'ont déclarée incorrecte, l'ordre canonique, plus transparent, a été restitué (que Philippe a rencontrée) ou on a fait du relatif un sujet (qui a rencontré Philippe). La complexité des phrases 19 et 22 est sans doute due à la structure possessive, intégrée en outre dans une structure présentative dans le cas de 19. Pour être rectifiée, la phrase 18 demandait qu'on donne un sens précis à que (qui n'établit qu'un lien vague entre l'antécédent et la relative : 'une langue à propos de laquelle je peux dire : je suis à l'aise') et l'on peut hésiter entre une notion locative (dans laquelle) ou instrumentale (avec laquelle). Dans 23, c'est probablement l'expression figée ça veut dire qui a masqué la nature résomptive du démonstratif. Nous reviendrons sur 1 et 25 plus loin. ${ }^{14}$

\subsection{Résultats spécifiques}

Malgré toutes les réserves qui précèdent, le test a prouvé son utilité. Nos hypothèses ont été confirmées. Le TJG a donné des informations plus étendues et bien plus précises que les productions libres sur l'appropriation des stratégies de relativisation du français par les étudiants. Cela est apparu en particulier quand ils ont eu à réagir à des relatifs plus rares comme dont ou à des relatifs en syntagme prépositionnel. Un bon nombre de sujets ont montré une uniformité de jugement pour une majorité d'items, ce qui prouve que la structure représentée dans la phrase est acquise ou en voie l'être, si les jugements sont conformes à la norme. Il y a eu cependant une variabilité de jugement importante pour quelques phrases, ou dans administration à l'autre du même test pour le second groupe, mais aussi dans les corrections proposées pour les jugements de types (3) et (4). Dans les cas de plus grande variabilité, en comparant les systèmes de relativisation en présence (voir Section 2), on peut détecter une attraction vers les structures de la L1. Cela a résulté en des jugements positifs qnand ils devaient être négatifs et inversement. Nous commenterons maintenant les résultats les plus significatifs concernant la compétence des sujets.

1. Le que relatif universel, le cousin de $\pi o v$, reste encore souvent accepté, jusqu'en dernière année d'études, comme équivalent de tout autre relatif. Entre $22 \%$ et $69 \%$ des sujets, selon la phrase, se satisfont de ce relatif passe-partout quand il leur est proposé (Tableau 6). Ces sujets continuent donc à utiliser la stratégie de relativisation sans trou syntaxique ni mouvement, étrangère au français standard, soit le système (1) de relativisation. On peut voir dans l'emploi persistant de cette stratégie aussi bien l'influence de la L1 (grec chypriote/grec commun) que celle de la grammaire universelle, le système (1) étant une stratégie de relativisation fort répandue dans les langues du monde.

\begin{tabular}{|l|l|l|}
\hline \multicolumn{1}{|c|}{ Phrase agrammaticale } & que en fonction de $\ldots$ & \multicolumn{1}{|c|}{$\begin{array}{c}\text { Taux } \\
\text { d'acceptation }\end{array}$} \\
\hline $\begin{array}{l}\text { *2. L'auteur mentionne certains tabous qu'ils existent à } \\
\text { propos des dialectes. }\end{array}$ & sujet & $25 \%$ \\
\hline $\begin{array}{l}\text { *26. Ils parlent le catalan que, pour eux, est une langue } \\
\text { maternelle ou une langue seconde. }\end{array}$ & sujet & $31 \%$ \\
\hline $\begin{array}{l}\text { *18. Le dialecte, c'est la langue du coeur, la langue qu'on } \\
\text { se sent à l'aise. }\end{array}$ & locatif & $69 \%$ \\
\hline $\begin{array}{l}\text { *15. La prononciation dépend de la région que le locuteur } \\
\text { vient. }\end{array}$ & locatif (provenance) & $47 \%$ \\
\hline $\begin{array}{l}* 16 . \text { Je viens d'acheter un nouveau manteau que j'en suis } \\
\text { très contente. }\end{array}$ & SPrép & $50 \%$ \\
\hline
\end{tabular}




\begin{tabular}{|l|l|l|}
\hline $\begin{array}{l}\text { *13. La porte que j'ai perdu la clé est celle de la cuisine, } \\
\text { pas celle du garage. }\end{array}$ & SPrép-génitif & $28 \%$ \\
\hline$* 27$. C'est un pays que son climat me plaît beaucoup. & SPrép-génitif & $41 \%$ \\
\hline
\end{tabular}

Tableau 6 - Acceptation de que relativiseur-complementiseur "neutre" $(N=32)$

2. Dans les productions libres des étudiants testés, comme nous l'avons fait remarquer plus haut, nous avons trouvé peu de relatives à résomptif. Par contre, dans la tâche de jugement de grammaticalité, ces relatives ont souvent été déclarées grammaticales ou acceptées (cf. Tableau 7, où les résomptifs sont soulignés).

\begin{tabular}{|l|l|l|}
\hline \multicolumn{1}{|c|}{ Phrase agrammaticale } & $\begin{array}{c}\text { Fonction du } \\
\text { résomptif }\end{array}$ & \multicolumn{1}{|c|}{$\begin{array}{c}\text { Taux } \\
\text { d'acceptation }\end{array}$} \\
\hline $\begin{array}{l}\text { *2. L'auteur mentionne certains tabous qu' } \text { 'ils existent à propos des } \\
\text { dialectes. }\end{array}$ & sujet & $25 \%$ \\
\hline $\begin{array}{l}* 23 . \text { Prenez par exemple le mot doll qu'en anglais ça veut dire } \\
\text { poupée. }\end{array}$ & sujet & $66 \%$ \\
\hline $\begin{array}{l}* 7 . \text { Hier soir il y avait à la télévision un film qu'Anna voulait } \\
\text { absolument le voir. }\end{array}$ & objet direct & $31 \%$ \\
\hline $\begin{array}{l}* 11 \text {. La copine à qui tu lui as prêté 50 euros, est-ce qu'elle te rendra } \\
\text { cet argent? }\end{array}$ & datif & $25 \%$ \\
\hline $\begin{array}{l}* 16 . \text { Je viens d'acheter un nouveau manteau que j'en suis très } \\
\text { contente. }\end{array}$ & objet indirect & $56 \%$ \\
\hline$* 22$. Connais-tu le garçon dont sa soeur sort avec Pascal ? & génitif & $72 \%$ \\
\hline
\end{tabular}

Tableau 7 - Exemples de relatives résomptives (incorrectes) acceptées par les sujets testés $(N=32)$

Gass (1979) a montré que les apprenants sont plus tolérants envers les résomptifs si leur langue en admet. Notre test semblerait le confirmer. Notez cependant que les résomptifs sont admis par nos sujets pour tous les syntagmes nominaux relativisables, y compris les syntagmes nominaux en fonction de sujet (ce qui n'est pas admis en grec). Dans l'interlangue des sujets qui acceptent les phrases ci-dessus, il semble qu'il $\mathrm{y}$ ait dans tout processus de relativisation en système (1), une place vide où peut apparaître facultativement un résomptif (cf. Hawkins \& Chan 1997 pour des faits similaires dans l'interlangue d'étudiants cantonais apprenants d'anglais). Il resterait à déterminer quels facteurs encouragent des jugements positifs en faveur de la résomption. Si la L1 a une influence, les positions les plus favorables à la présence d'un pronom/adverbe de reprise seraient celles des niveaux les plus bas sur l'échelle d'accessibilité de Keenan et Comrie (1977), illustrées ici dans les phrases 16 et 22. Mais il faudra enrichir le TJG d'items supplémentaires avec résomptif pour tester cette hypothèse.

3. Comme déjà les productions libres le montraient, la différence entre qui et que n'est pas complètement acquise par tous les étudiants. Il semble que parfois, qui et que soient tenus pour interchangeables en tant que relativiseurs-complémentiseurs. Considérez (6) et (7), où $\mathrm{N}=32$.

(7) (a) 2. *L'auteur mentionne certains tabous qu'ils existent à propos des dialectes (25\% acceptent)

(b) $26 . *$ Ils parlent le catalan que, pour eux, est une langue maternelle ou une langue seconde. ( $31 \%$ acceptent)

(c) 9 . Il connaît plusieurs langues qui sont parlées en Russie. (22\% condamnent et proposent que)

(8) (a) 6. La femme qu'a rencontrée Philippe était la directrice de l'entreprise. (34\% acceptent, les autres corrigent en changeant l'ordre des mots, ou en proposant qui et en modifiant l'accord du participe)

(b) 21 . Voilà les preuves que je cherchais à obtenir. ( $85 \%$ acceptent) 
Cette situation remet-elle en cause l'applicabilité de la hiérarchie typologique de Keenan et Comrie (1977) au domaine de l'acquisition des langues étrangères ? La théorie du marquage dans le progrès de l'apprentissage des relatives qu'on a fondée sur cette hiérarchie prédirait en principe que qui, le relatif sujet, donc le moins marqué, devrait être déjà bien acquis par les étudiants avancés. Elle prédirait d'autre part que que, COD dans les phrases 6 et 21, devrait être jugé également acceptable, ce qui n'est pas le cas puisque la présence ou l'absence de l'inversion «stylistique» verbe-sujet entraîne un jugement radicalement différent. Cela apporte certes de l'eau au moulin de ceux qui s'opposent à l'utilisation de la HA pour expliquer l'apprentissage des relatifs, qui préfèrent travailler dans le cadre des configurations syntaxiques et pour lesquels la distance/proximité du relatif par rapport au site relativisé explique les difficultés des appprenants dans la production des pronoms relatifs (concernant ce débat voir Eckman 2007, Hawkins J. 2007, Hawkins R. 2007). Or la configuration syntaxique explique difficilement que (7c) ne soit pas jugé acceptable. Doit-on invoquer hiérarchies et configurations pour comprendre les confusions que font certains de nos sujets entre qui et que? Il semble que dans leur interlangue les deux formes soient les variantes libres (?) d'un complémentiseur universel qui sert simplement à connecter un nom à une proposition applicable à ce nom (Système (1)). Le système (2) (grec commun soutenu), qui pourrait entraîner des tranferts positifs dans l'acquisition du relatif français et où la HA et les configurations syntaxiques ont leur rôle à jouer, ne semble pas avoir toute l'influence que les professeurs de FLE souhaiteraient qu'il ait ${ }^{15}$.

4. Dans leurs productions libres les étudiants n'ont quasiment jamais employé le pronom qui dans un syntagme prépositionnel et lui ont préféré lequel. Cela est confirmé par leurs corrections des phrases en (9).

(9) 1. Je me souviens bien de Philippe Leblanc avec qui j'étais soldat en Algérie.

( $47 \%$ condamnent, dont la moitié corrigent avec lequel)

25. Donne-moi la liste des gens à qui tu as envoyé des invitations.

(53\% condamnent, dont $40 \%$ corrigent avec auxquels).

On peut voir dans cette préférence du relatif fléchi lequel une possible influence du relatif $o$ oлoíoৎ luimême fléchi (Système (2)). On observe une tendance similaire sporadique pour qui (sujet). Considérez en (10) les corrections proposées pour les phrases 26 et 10.

(10) 26. [...] le catalan, laquelle est pour eux une langue maternelle ou une langue seconde.

10. [...] la célèbre actrice laquelle je n'ai pas besoin de vous présenter.

Cet emploi de lequel hors Syntagme Prépositionnel, qui n'est pas grammaticalement incorrect en français, est pragmatiquement improbable dans les phrases proposées. On peut soupçonner ici des effets d'hypercorrection provoqués par le système (2). La sur-spécificité morphosyntaxique de $o$ oлoíoৎ du grec soutenu peut favoriser l'emploi de lequel, les deux formes étant des calques l'une de l'autre. Dans quelles conditions cette possibilité est-elle réalisée ? Cela reste à découvrir. Il n'en reste pas moins que même si les transferts en français viennent de façon prépondérante du système (1), le moins marqué morphosyntaxiquement, le système (2), le plus marqué, peut avoir à l'occasion certains effets.

\section{Conclusion}

Le test décrit ici nous fait mesurer les difficultés que l'apprenant chypriote rencontre dans l'appropriation de la relativisation en français, et aussi celle du chercheur-enseignant qui essaie de s'expliquer et d'expliquer comment se fait cette appropriation. Du point de vue de l'apprenant, la difficulté vient d'abord de la situation linguistique qui est son quotidien. Pour lui, la langue source est double, constituée par deux variétés clairement différenciées. En second lieu, du point de vue des stratégies de relativisation, les deux variétés sont radicalement différentes : a) pour la variété vernaculaire, un système à particule invariable, avec résomption facultative ; b) pour la variété haute, un système avec flexion morphologique maximale pour le genre, le nombre et le cas. Le TJG a montré que la variété haute ne semble pas avoir 
une forte influence sur l'interlangue des apprenants. Son système (2) de relativisation, qui se rapproche de celui du français standard par sa complexité morphosyntaxique, pourrait faciliter l'apprentissage (Monville-Burston et Kounouni 2007). Pourtant, si l'apprenant en fait usage, le succès n'est pas toujours garanti. L'influence de ce système est réelle cependant, puisque des hypercorrections émergent de temps en temps, qui la manifestent. Le système (1), au contraire, est dominant et source de transferts fréquents dans l'interlangue, comme l'a montré le TJG : confusions entre que et qui, acceptation du que universel pour toutes les fonctions du relatif et des pronoms de reprise. Il existe une insécurité linguistique due à l'alternance entre les deux codes ('grec du dimanche' et 'grec de tous les jours') qui reste à explorer plus avant. Cette insécurité se répercute dans la relativisation pour la langue cible, d'où la variabilité dans les jugements pour certains items du TJG et l'évitement des relatifs 'difficiles' dans les productions libres. Du point de vue du chercheur, il sera nécessaire de perfectionner le TJG, pour mieux l'adapter aux traits de l'interlangue que sa première version a mis en lumière, ceux qui ont été discutés en 3.4. En particulier, il faudra le subdiviser pour tester ces traits de façon plus systématique et bien sûr continuer à l'utiliser conjointement avec d'autres instruments de recherche, corpora et tests différents.

\section{Références bibliographiques}

Arvaniti, A. (2006). Linguistic practices in Cyprus and the emergence of Cypriot Standard Greek. San Diego Linguistic Papers 2, 1-24.

Blanche-Benveniste, C. (2000). Approches de la langue parlée en français. Paris : Ophrys.

(2003). La langue parlée, in Marina Yaguello (dir.), Le grand livre de la langue française. Paris : Seuil. $317-$ 344.

Bley-Vroman, R, Felix, S. \& Ioup, G. (1988). The accessibility of universal grammar in adult language learning. Second Language Research 4, 1-32.

Chaudron, C. (1983) Research on metalinguistic judgments : A review of theory, methods and results. Language Learning 33, 343-377.

Creissels, D. (1995). Éléments de syntaxe générale. Paris : PUF.

Cowan, R. \& Hatasa,Y. A. (1994). Investigating the validity and reliability of native speaker and second-language leraner judgments about sentences. In Tarone, E., Gass, S. M., \& Cohen, A. (dir.), 287-302.

Damourette, J. and E. Pichon. (1911-1934). Des mots à la pensée. Essai de grammaire de la langue française, tome 4. Paris : d'Artrey.

Davies, W. D. \& Kaplan, T.I. (1998). Native speaker vs. L2 learner grammaticality judgments. Applied Linguistics $19,183-203$.

Delofeu, J. (1981). Perspective linguistique et sociolinguistique dans l'étude des relatives en français. Recherches sur le français parlé 3, 134-193.

Doughty, C. (1991). Second language instruction does make a difference. Evidence form an empirical study of SL relativization. Studies in Second Language Acquisition 13, 431-469.

Eckman, F., L. Bell and D. Nelson. (1988). On the generalisation of relative clause instruction in the acquisition of English as a second language. Applied Linguistics $9: 1,1-20$.

- (2007). Hypotheses and methods in second language acquisition : Testing the noun phrase accessibility hierarchy on relative clauses. Studies in Second Language Acquisition 29, 321-327.

Ellis, R. (1991) Grammaticality judgements and second language acquisition. Studies in Second Language Acquisition 13,161-186.

Gadet, F. (1989). La langue ordinaire. Paris : Armand Colin. (1995). Les relatives non standard en français parlé, le système et l'usage. Etudes Romanes 34. 141-162. (2003). La relative française, difficile et complexe, in Sibylle Kriegel (dir.), Grammaticalisation et réanalyse Approches de la variation créole et française. Paris : CNRS Éditions, 251-268.

Gass, S. M. (1979) Language transfer and universal grammatical relations. Language Learning 29 : 2, 327-344. (1994) The reliability of second-language grammaticality judgments. In Tarone, E., Gass, S. M., \& Cohen, A. (dir.), 303-322.

Godard, D. (1988). La syntaxe des relatives en français. Paris : Éditions du CNRS.

Goss, N, Ying-Hua, Z \& J. Lantolf. (1994). Two heads may be better than one : Mental activity in second-language grammaticality judgments, in E. Tarone et al., 263-286.

Hancock, V. \& N. Kirchmeyer. (2002). A la recherche des traits d'une organisation discursive avancée en français L2: la relative aux micro- et macro-niveaux dans un corpus d'apprenants. L'Information grammaticale 93, 3-9. 
— (2005). Discourse structuring in advanced L2 French : The relative clause, in J.-M. Dewaele (dir.), Focus on French as a foreign language-Multidisciplinary approaches. Clevedon : Multilingual Matters.17-35.

Hawkins, J. (2007). Acquisition of relative clauses in relation to language universals. Studies in Second Language Acquisition 29, 337-344.

Hawkins, R. (1989). Do second language learners acquire restrictive relative clauses on the basis of relational or configurational information? The acquisition of French subject, direct object and genitive relative clauses by second language learners. Second Language Research 5, 156-188.

- (2007). The noun phrase accessibility hierachy : lame duck or dead duck in theories of SLA ? Studies in Second Language Acquisition 29, 345-359.

Holton, D., P. Mackridge et I. Philippaki-Warburton (1997). Greek: A Comprehensive Grammar of the Modern Greek Language. London : Routledge.

Izumi, S. (2003). Processing difficulty in comprehension and production of relative clauses by learners of English as a second language. Language Learning 53 : 2, 285-323.

Johnson, J., K. Shenkman, E. Newport \& D. Medin. (1996). Indeterminacy in the grammar of adult language learners. Journal of Memory and Language 35, 335-352.

Karyolemou, M. et P. Pavlou. (2001). Language attitudes and assessment in a bi-dialectal speech community. Proceedings of the First International Conference on Language Variation in Europe. Barcelone : Universitat Pompeu Fabra. 110-120.

Keenan, E. et B. Comrie. (1977). Noun phrase accessibility and universal grammar. Linguistic Inquiry, 8 : 1, 63-99.

Mackridge, P. (1985). The Modern Greek language. Oxford : Clarendon Press.

Mandell, P. (1999). On the reliability of grammaticality judgement tests in second language acquisition research. Second Language Research $15:$ 1, 73-79.

Monville-Burston, M. et M. Kounouni (2007). Deux procédures expérimentales pour tester la relativisation dans l'interlangue d'apprenants chypriotes hellénophoihnes en FLE. Communication présentée au $14^{\text {ème }}$ colloque international de GALA (Greek Applied Linguistics Association), 14-16 déc., Université de Salonique.

Monville-Burston, M. et F. Doa (à paraître). Aspects of the interlanguage of advanced Greek-speaking Cypriot learners of French : Relative clauses. In E. Labeau et F. Myles (dir.), The advanced learner variety : The case of French, Berne : Peter Lang, 125-147.

Mirambel, A. (1939). Précis de grammaire élémentaire du grec moderne. Paris : Les Belles Lettres.

Nicholas, N. (1998). The story of pu. The grammaticalisation in space and time of a Modern Greek complementiser. Thèse de doctorat, The University of Melbourne.

Tarone, E., Gass, S. M., et Cohen, A. (dir.) (1994). Research methodology in second language acquisition. Hillsdale, NJ : Lawrence Erlbaum.

Triandaphyllidis, M. (2003) Petite Grammaire du grec moderne. Traduit par F. Duisit et O. Merlier, $2^{\text {ème }}$ éd., Thessalonique : Université Aristote.

Wilmet, M. (2003). Grammaire critique du français, $3^{\text {ème }}$ éd.. Bruxelles: De Boeck \& Larcier, Duculot.

\footnotetext{
${ }^{1}$ Sur la diglossie chypriote, voir par exemple Karyolemou \& Pavlou (2001) et Arvaniti (2006). Il est à noter que la situation diglossique se complique de la présence de l'anglais, langue coloniale qui reste largement utilisée dans certains domaines de l'adminitration et dans les nombreuses écoles privées, et dont l'influence est sensible sur le chypriote. Les variétés haute (grec commun) et basse (grec chypriote) ne sont pas mutuellement intelligibles, mais les Chypriotes, apprenant le grec commun à l'école comprennent les Grecs, tandis que l'inverse n'est pas vrai.

${ }^{2}$ Sur la relativisation en grec, voir Mirambel 1939, Mackridge 1985, Holton et al. 1997, Triandaphyllidis 2003.

${ }^{3}$ On peut consulter des grammaires de référence ou des ouvrages comme Godard 1988, Creissels 1995, Wilmet 2003, pour compléter cette présentation en raccourci du relatif français.

${ }^{4}$ Suivant la terminologie de Delofeu (1981).

${ }^{5}$ Il s'agit ici du français normalisé. Le relatif dans le français vernaculaire (« familière/populaire ») partage les traits du système (1) grec-chypriote/grec familier. Il a été décrit par Damourette et Pichon (1911-1934), Delofeu (1981), Gadet (1989, 1995, 2003), Blanche-Benveniste (2000, 2003), entre autres.
} 
${ }^{6}$ Delofeu (1981 : 186) parle d'un système «bricolé », manipulé par les grammairiens dirigistes du $17^{\text {ème }}$ siècle.

${ }^{7}$ Pour une plus ample discussion de cette question et pour une étude à orientation contrastive, voir Monville-Burston et Doa (à paraître).

${ }^{8}$ Voir aussi la remarque de Delofeu $(1981: 188)$ sur la fréquence d'emploi des divers relatifs français : « le standard n'est pas utilisable dans les énoncés informatifs [= non-littéraires]; les formes correspondantes non standard sont 'tabou'. La solution consiste à ne produire que des relatives qui soient à la fois admises par l'école et formées selon les règles du vernaculaire : on ne fera que des relatives en qui sujet et que objet. »

${ }^{9}$ Dans un autre instrument de recherche (test de traduction), appliqué en $3^{\text {ème }}$ année et destiné lui aussi à étudier la relativisation chez nos étudiants, $66 \%$ des réponses fautives contenaient un que relativiseur-complémentiseur (Voir Monville-Burston et Kounouni (2007).

${ }^{10}$ Voir Monville-Burston et Kounouni (2007).

11 «Such a position is tantamount to saying that GJ [grammatical judgement] tasks are only going to provide valid and reliable data when the subjects being tested have attained sufficient proficiency in the L2 (where defining 'sufficient proficiency' then becomes the important issue). » Le fait que certains items ont provoqué des réponses très variables laisserait alors croire que pour ces items (items 12, 22, 23), la compétence langagière du groupe sur la relativisation n'était pas 'suffisante', où 'suffisante' resterait à définir plus précisément.

${ }^{12}$ La première administration du TJG à 20 sujets au printemps 2007 a été considérée comme un essai. La seconde en a été double : en novembre 2007 à 12 sujets puis à nouveau en avril 2008 au même groupe. Malheureusement 3 étudiants du groupe ayant terminé leurs études, ils n'ont pas pu être retestés. Nous n'avons donc en mains que neuf paires de tests.

${ }^{13}$ Il reste à faire des analyses plus fines. Par exemple, certains étudiants sont plus constants que d'autres et certaines phrases sont plus marquées que d'autres par la variabilité. D'autre part les phrases qui ont été jugées incorrectes n'ont pas été forcément corrigées de la même façon dans les deux administrations successives du test.

${ }^{14}$ Les phrases 19 et 23 demanderont à être réexaminées avant d'être inclues dans la version 2 du TJG, pour s'assurer qu'elles ne testent pas autre chose que la relativisation.

${ }^{15}$ Voir Monville-Burston et Kounouni (2007). Si l'on fait traduire en français à des étudiants chypriotes (niveau B1B2) des propositions relatives contenant le pronom o oлoio (Système (2)), on obtient des résultats meilleurs (amélioration de $25 \%$ environ) que si on donne comme point de départ une relative contenant $\pi$ ov (Système (1)). 